\title{
MCDM Approach to Improve the Quality of Broadcasting Technique in Wireless Ad-hoc Networks
}

NAEEM AHMAD ( $\nabla$ naeem08ahmad@gmail.com )

Madanapalle Institute of Technology and Science https://orcid.org/0000-0003-4627-6400

Shuchi Sethi

Jamia Millia Islamia Central University: Jamia Millia Islamia

Md Gulzarul Hasan

Madanapalle Institute of Technology and Science

Rejaul Karim Barbhuiya

National Council of Educational Research and Training

Research Article

Keywords: Broadcast, Flooding,MCDM, WANETs, AHP

Posted Date: April 13th, 2021

DOI: https://doi.org/10.21203/rs.3.rs-357531/v1

License: (c) (i) This work is licensed under a Creative Commons Attribution 4.0 International License.

Read Full License 


\title{
MCDM approach to improve the quality of broadcasting technique in Wireless Ad-hoc Networks
}

\author{
Naeem Ahmad ${ }^{*, 1}$. Shuchi Sethi ${ }^{2}$. \\ Md. Gulzarul Hasan ${ }^{3}$. Rejaul Karim Barbhuiya ${ }^{4}$
}

the date of receipt and acceptance should be inserted later

\begin{abstract}
Contemporary research work in the field of Wireless Ad-hoc Networks (WANETs) are focused on most important issue of performance, minimize the congestion in the network. A number of research proposals have come up with innovative query transmission approaches in order to scale down congestion, route latency and energy consumption to the feasible extent. It has been shown experimentally that these approaches have mainly focused on checking propagation of route query using query broadcast repealing technique which unfortunately paves way to additional routing overhead. This leads to increased energy-consumption which offsets the benefits. This paper introduces a self-aware query-broadcast approach which is dynamic in nature and obtains desirable path in discovery phase without counterbalancing the advantages reaped from confining the request zones. The proposed approach relies on Analytic Hierarchy Process (AHP), which utilizes the routing metrics in estimating the weighing factors for combined weight metrics. For evaluation of the proposed technique, critical and analytical assessment is done in terms of query-diffusion, packet-delivery ratio and route-latency. The results reveal the proposed approach is ideal, optimal and highly practical for application in WANETs.
\end{abstract}

Keywords Broadcast · Flooding · MCDM · WANETs · AHP.

\section{Introduction}

A non-static networks of mobile nodes without any co-ordination of infrastructure form WANETs. Delay in discovery of route along with repeated transmission of query have been identified as major challenges in these networks. While inability to find route rapidly renders it old, retransmissions cause network congestion. Striving the balance between route-discovery and retransmission is even

1 Department of Computer Applications

Madanapalle Institute of Technology and Science

Madanapalle,A.P.,India

E-mail: naeem08ahmad@gmail.com

- 2 Department of Computer Science, Jamia Millia Islamia(A Central University)

Jamia Nagar,New Delhi,India

E-mail: shuchi.sethi@yahoo.com

. 3 Department of Mathematics, Madanapalle Institute of Technology and Science

Madanapalle,A.P.,India

E-mail: gulzarhsn@gmail.com

- 4 Central Institute of Educational Technology, National Council of Educational Research and Training (NCERT)

New Delhi,India

E-mail: rejaul.ncert@nic.in 
bigger challenge. Discussion of revoking approaches that can contribute to bring overheads down have been presented by the authors in [1]. First controlled broadcasting technique,TTL-sequenced based broadcasting, proposes node at source to repeatedly transmit query for locating route as attempts keep failing. Subsequently, a broadcast-repealing technique, Blocking Expanding Ring Search (BERS)[16] was proposed that deliberately induced delay in the processing of the query to send the stop - instruction packet issued by source-node to chase the query-packet. This reduces query-broadcast. Based on this broadcast-repealing strategy, many other approaches were deployed to decrease the extra cost associated with finding the route [1].

As we are familiar that rebroadcasting is the process of repeated broadcasting as attempt to deliver fails. In route-discovery, intermediate nodes forward the query, to travel the network based on the predefined TTL-value. The nodes in-between drop the query when counter reaches its predefined TTL limit. This can have a side effect and gives rise to issue called destinationunreachable and also requires intimating the source node about the same. Source then rebroadcasts the route-query with a new increased TTL limit. Rebroadcasting deals with the flooding for search at least possible cost, while it leads to longer routing delay. It is observed that TTL-sequenced based ERS circulates the query over and over in the network. Authors in different literatures $[16,20,5,10]$ also points out TTL-sequence is inefficient in terms of energy usage. Research effort has been made to improve TTL-sequence approach $[1,2]$. The techniques have limitations of re-broadcasting and larger request-zones where query carrying request circulates with additional cost of query-chasing. A cache aware technique tries to address it in [19] based on educated guess that the new route will contain nodes from most recently used route. This approach would yield better results in terms of smaller request zone. Query Localization Technique (QLT) [19] is one of such techniques that makes use of old-route information to find a new route. The source node keeps the initial counter value 0 in the header of query and broadcasts the query containing set of old route nodes. To find new route, each node is visited to verify validity of the route and in case of invalid route, current node triggers the process of route-reconstruction. The node increments a counter placed in header of the query to continue the forwarding. This pattern of discovery raises 2 important questions for further investigation: (1)Will route query be able to reach its destination? (2) What will be the implications if source node uses the approach of rebroadcasting to discover the route?

The problem arose in the said questions is known as destination-unreachable problem. All context-aware broadcasting techniques face challenges in discovering destination especially under 2 circumstances:i) if participating nodes are new and ii) destination node has moved from its previous location. Figure 1(a,b) explains this fact. To counter the issue, context-aware broadcasting techniques follow the TTL sequence-based broadcast that is very expensive as discussed above. Various research efforts have been done to address the destination unreachable problem in routediscovery by designating a local region small enough to make the attempt successful. This paper is an effort to address the broadcasting overheads and present the approach for finding a tolerable request-zone for query-broadcast at least overheads. A similar approach is proposed to overcome the overheads in context-aware broadcasting techniques by avoiding repeated broadcast due to unreachable problem. Proposed approach provides small enough and highly predicted request zone, which is more probabilistic and time-efficient than other context-aware broadcasting techniques. To make it more probabilistic, a marginal cost is applied on search extent to choose the tolerable search region in others. This marginal cost is defined as multi-criteria decision making (MCDM) process to estimate the weights. The defined process is solved using well-known method Analytical Hierarchical Process (AHP). Results are obtained in terms of query-diffusion, packet-delivery ratio and routing delay, which is compared with other approaches including QLT[19], AODV-TTL [18] and BERS+ [5]. Obtained results shown that proposed approach outperforms and significant reduction in the broadcasting overheads is observed. 


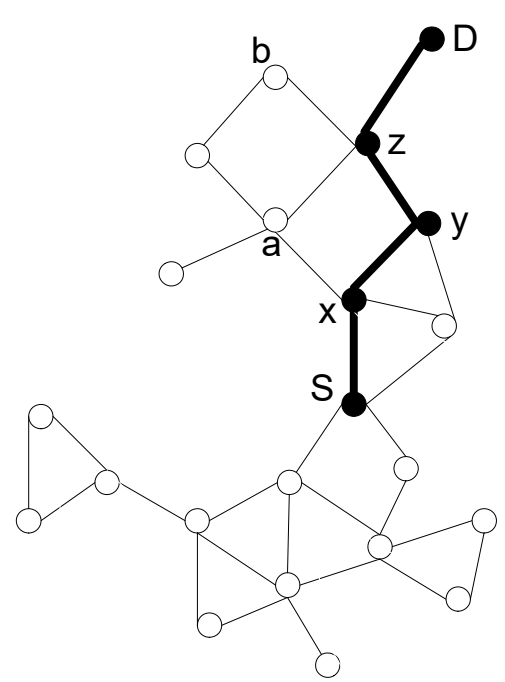

(a)

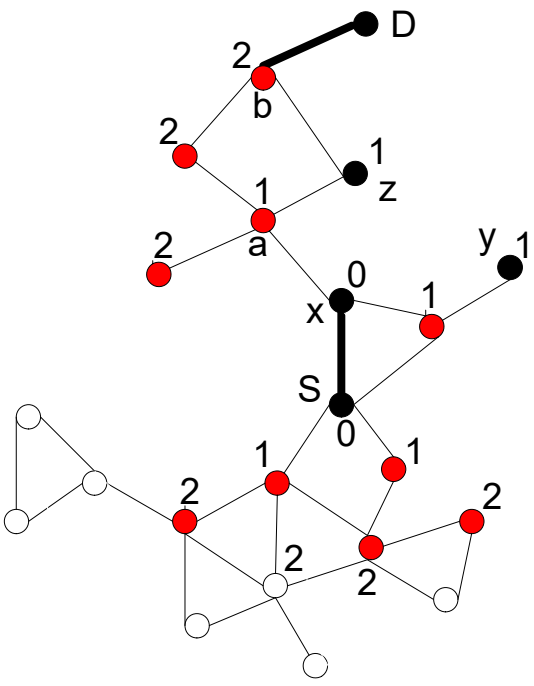

(b)

Fig. 1: (a)Most recently used route. (b)Failed attempt of route discovery when target node or any of the old route-nodes moves to new location.

\section{Related Contributions}

There are many approaches to discover route in WANETs, most common being reactive routing with most significant disadvantage of continued propagation of query even after route had been discovered. This leads to undesirable consequences like congestion in network, broadcast storm [3]. To overcome the issue many researchers have proposed dividing the network into small and manageable request zones for intermediate nodes. On basis of approach of defining these zones, query broadcasting techniques can be categorized as: Controlled query broadcasting techniques and context-aware query broadcasting techniques.

The categorization is based on use of routing-cache in picking request-zone is detailed in Figure 2. Control based methods rely on flooding without having previous routing context, which is shown in red colored texts in Figure 2. This means that previous communication is not taken into consideration during the broadcasting process. In early phase of AODV development as in [18], AODV has traditionally applied TTL-sequence based repeated query broadcast [6]. In similar manner, improvised strategies were proposed in $[5,16,21]$. To address the issue of repeated broadcast, ERS, LHBA found the use of chase packet to annul the broadcast query. First time, this technique used stop-instruction packets to cancel the query propagation in the network. The approach pioneers in deploying stop-instruction packet to cancel the query broadcast. On discovering the route, contrary to conventional approach of destination replying as unicast to source, reply is broadcasted. This gives us twofold functionality where reply packet acts as reply as well as chase packet. These stop-instruction packet is issued at either source-node or destination node to break off the query-broadcast after successful route-discovery. Due to its limited search area, its benefits are also limited and still suffers with broadcast storm problem [9]. A few advanced versions like BERS [16] and BERS* [20] have been proposed. Although gain is imminent in terms of energy savings, techniques add to route-latency due to added delay in query processing at intermediate nodes. Besides these are not adaptive to mobility of targets leading to repeated broadcast due to limited journey of route-queries. 


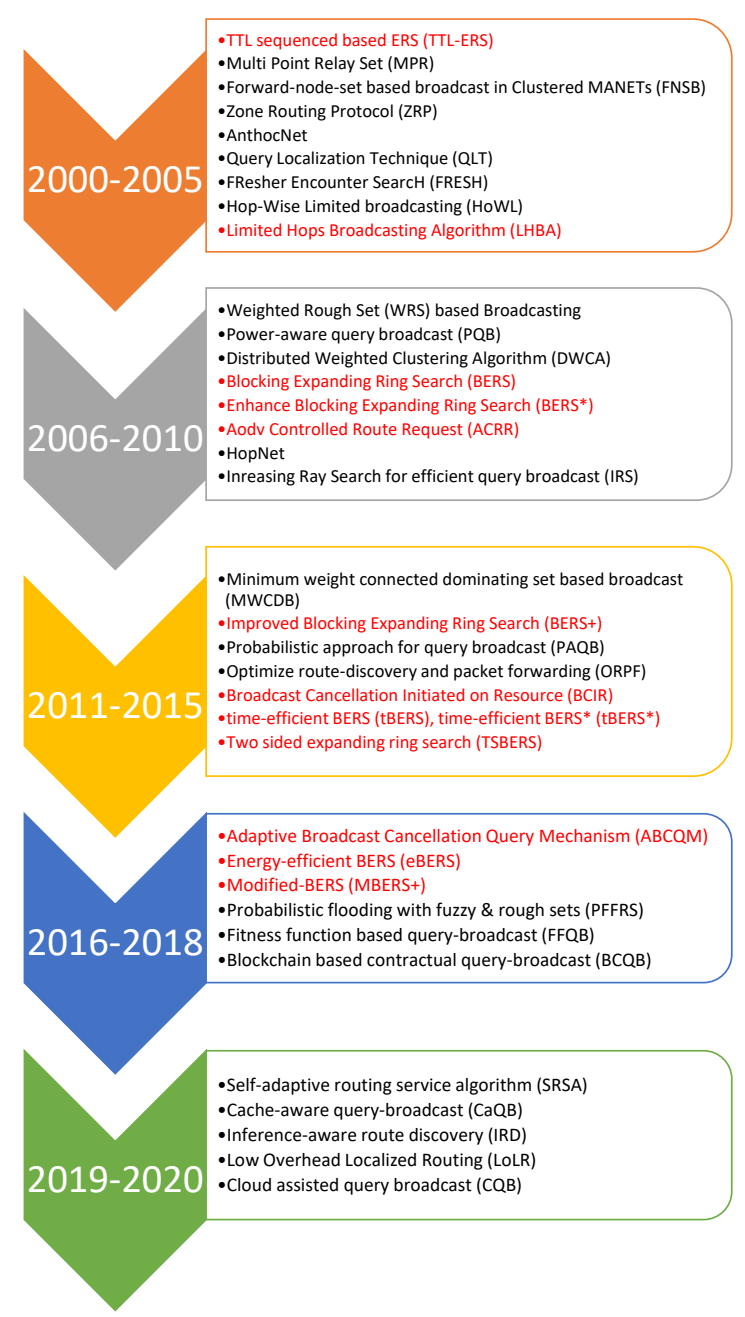

Fig. 2: Classification of query-broadcast methods (Please see full abbreviations in appendix A)

To achieve quick adaptation to mobility, BERS+ [5] and MBERS+ [10] have been proposed which are the most recent contributions. Time and energy parameters have been considered making algorithms. BERS+ enables the route-query to travel beyond the predefined request-zone making it a two tier approach. The search-regions inside the predefined request-zone is referred as first tier and outside being the second tier. In first tier, query moves freely without any processing delay waiting for chase-packet thus speeding up the route-discovery. It adds processing delay in second tier to help chase-packet in coping the query-broadcast. This minimizes an end to end delay and maximizes the reception ratio. In BERS+, source-node is responsible for issuing the chase-packets after discovering the route. MBERS+ made replying and chasing events concurrent to speed up the chasing by issuing chase-packets at destination-node. Although these algorithms are currently found in applications, all these techniques are very inefficient for small to moderate network size where issuing chase packet is again a broadcast-overhead. This area requires lot of research efforts.

With increased variety of devices came the notion of grouping the devices giving rise to cluster based algorithms. WCA [15], PWCA [17] and DWCA [13] unveiled the idea of device clustering with master node formation to carry out smooth routing within clusters. The approach proved to 
be highly effective over conventional ones in controlling the broadcast and reducing the forwarding rate by way of permissions. But there was lot of room for effort in controlling the query broadcast. Therefore cluster-based routing methods are extended to use the controlled-query broadcast features [10]. This brought limitations of controlled flooding back again.

The second category, context-aware broadcasting approaches rely on available routing cache to estimate the desirable route, which is shown in black colored texts in Figure 2. Putting old route to use from the routing cache helps in deciding which nodes to pick as forwarding nodes. It also permits the routing limited to the search-region. These techniques control the broadcast expenses of route discovery process. WRS [23], and [24] are proposed based on routing cache which select the set of neighbour nodes based on a few metrics. Many more techniques are proposed on the similar line those are surveyed in recently publish literatures $[1,4]$. In the meanwhile, several power aware routing protocols have been proposed. The main idea of these protocols is to use residualenergy to improve the life of the network by balancing load among the intermediate nodes lying on the route $[14,25]$. Although these protocols reduce route-reconstruction problem, it may lead to longer route thus giving rise to less packet delivery ratio and longer routing delay. One such routing protocol in [26] is proposed, which uses fitness function to select the energy-efficient route from the available set of routes for the same communicating nodes. In other literature [27], authors extended routing to cloud environment in which cloud-assisted routing has been proposed. It is observed that route-maintenance procedure leads to longer route if route-break occurs. Here our focus is on addressing the routing overheads of route-discovery phase in WANETs. In recent years, researchers have worked on different aspects of wireless networks including security, shortest path, energy-efficient routing, however efficient query-broadcast is still required attention to address the problem of unreachability as we have observed in the related contributions.

In this paper, our contributions are listed as follows:

1. Most recent route of the routing cache is used, which guides in choosing the small enough and highly predicted request-zone. In order to counter the unreachability, two types of nodes are identified bridge the gape between old-route nodes and target node.

2. These primary nodes apply marginal cost to continue the query-forwarding thus extending the the request-zone to find the target node. This marginal cost is a combined weight metrics that uses mobility, node-density, node-status and energy.

3. Routing is a decision making process depending on criteria that includes performance metrics and node-attributes. Here, we defined combined weight metrics as a multi-criteria decision making process, which relies on AHP process in order to estimate the weights.

We refer whole approach as Self-aware query-Broadcast Approach (SBA). The proposed approach deals with dynamic request-zone by adopting AHP process, a simple MCDM method to counter the routing issues. The metrics used adopted model of marginal cost are arranged in the hierarchy to defined the MCDM process. The SBA utilizes the two-hops proximity information for marginal cost value to have the optimal request-zone.

\section{Self-aware query-Broadcast Approach (SBA)}

SBA is a context-aware query-broadcast approach for wireless networks. This work assumes that target mobile node has moved from previous location making the recently used route invalid. The proposed approach enhances the query-broadcast process of route-discovery in wireless networks utilizing recently used route and four routing metrics including mobility, energy, node-status and node-density. The primary nodes use two-hops away node information for the said routing metrics. The primary nodes issue beacons to retrieve the node information such as speed, buffer-queue length, node density etc. This beacon is issued by the nodes declare itself as primary nodes. On receiving beacons, the primary nodes compute average distances, average speed, and buffer-queue 


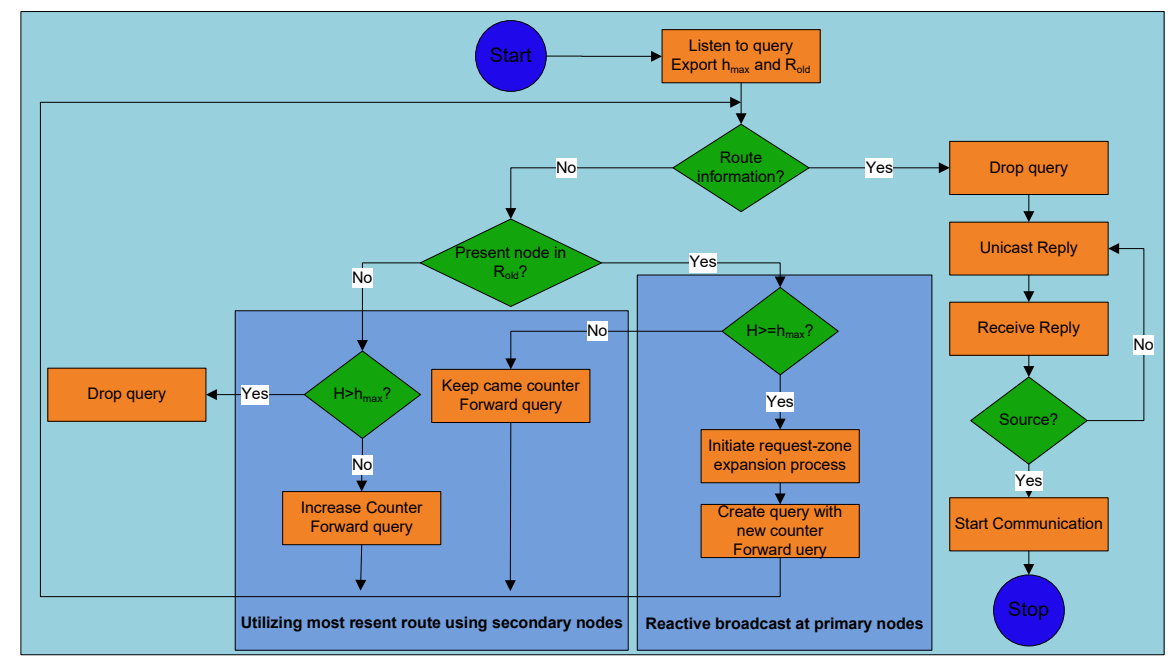

Fig. 3: Work flow of proposed approach

length. Afterwards AHP process utilizes these computed metrics to estimate the weights for the primary nodes during the request-zone expansion. Finally primary nodes continue to forward the query in this expanded request zone. Figure 3 shows work flow of the proposed approach and the process to compute the weights is discussed in Section 3.4.

\subsection{Metrics to predict the request-zone}

Optimal request-zone for query-broadcast is always desirable with predictable routing metrics. The decision of expanding request-zone based on a specific metric may lead to larger request-zone thus making inefficient query broadcast. There are many decision criteria that affect the route-discovery process. Therefore this section presents some of the routing metrics which have been considered for the selection of optimal request-zone.

\subsubsection{Mobility and its related information}

The mobility represents the movement of the node in the network, which depends on other metrics including distance, speed and variation. In our work, we have chosen relative mobility of adjacent nodes to compute the marginal cost. Let $\left(x_{i}, y_{i}\right)$ be the position coordinate of the $i^{\text {th }}$ proximity node and $\left(x_{c}, y_{c}\right)$ be the position coordinate of primary node, the distance between these nodes can be computed as :

$$
D=\sqrt{\left(x_{i}-x_{c}\right)^{2}+\left(y_{i}-y_{c}\right)^{2}}
$$

Primary nodes utilize the average distance of all proximity nodes for marginal cost function. Let $m$ be number of proximity nodes of the primary node, average distance between proximity nodes and primary node is:

$$
D_{a v g}=\frac{\sum_{i=1}^{m} \sqrt{\left(x_{i}-x_{c}\right)^{2}+\left(y_{i}-y_{c}\right)^{2}}}{m}
$$


Let $S_{i}$ be the speed of $i^{t h}$ mobile node from the proximity of the primary node. The average speed of the proximity nodes $S_{a v g}$ is computed as:

$$
S_{a v g}=\frac{\sum_{i=1}^{m} S_{i}}{m}
$$

Variation is also one of the important factor to avoid the speed outliers, which could contribute to the factor affecting the mobility. Larger variation in speed of moving nodes may lead to biasness in mobility. Let $V^{(i)}(t)$ represents variation in speed of proximity nodes of $i^{\text {th }}$ primary node at time $t$, variation can be computed as

$$
V^{(i)}(t)=\frac{\sqrt{\sum_{i=1}^{m}\left(S_{a v g}-S_{i}\right)}}{m-1}
$$

Where $S_{a v g}$ is the average speed of the proximity nodes given in Eq. 3.

\subsubsection{Tracking status of nodes}

A node can receive packets from different adjacent nodes. Each node in the network has limited buffer to avoid the congestion thus retaining these packets temporarily. Due to heavy traffic load, this limited buffer at node causes the packets-drops. Node-status represents this traffic load at each node, which is observed through beacons exchange. The average capacity of buffer-queue $\left(Q_{a v g}(t)\right)$ at node can be computed as:

$$
Q_{\text {avg }}(t)=\frac{\sum_{i=1}^{m}\left(Q_{\max }-Q_{i}(t)\right)}{m Q_{\max }}, \quad Q_{i}(t)>Q_{\min } \forall i \text { in } R
$$

Where $Q_{\max }$ is maximum threshold of queue capacity. In this simulation work, we have set buffer-queue at 50, which is threshold value for each node. Here it is assumed that $i^{\text {th }}$ node contains $Q_{i}(t)$ number of packets in buffer-queue at $t$. The buffer at each node must be able to contain at least $Q_{\min }$ number of packets to contribute to the marginal cost function. $R$ is number of nodes in neighbor table.

\subsubsection{Calculating energy}

Energy management is always desirable for longer life of the network. In order to get this, node with high energy is chosen to provide more stable route. Here, the term energy indicates energy-saving of battery operated mobile nodes in wireless ad hoc networks. The average energy-saving of the nodes $\left(E_{\text {avg }}(t)\right)$ at node can be computed as:

$$
E_{\text {avg }}(t)=\frac{\sum_{i=1}^{m}\left(E_{i}(t)\right)}{m}, \quad E_{i}(t)>E_{\text {min }} \forall i \text { in } R
$$

Where $E_{i}(t)$ is current energy of $i^{t h}$ node at $t$. In this simulation work, initial energy of each node is 1000 joules. We have set 20 joules as threshold value for each node, which is least energy (i.e. $\left.E_{\min }\right)$ to contribute to the marginal cost function. 


\subsubsection{Determining node-density}

The number of neighboring nodes denotes the node-density, which is also one of the important factors to optimize the request-zone for query-forwarding. High density leads to robust connectivity and helps to select reliable node for communication thus reducing the risk of unreachability. Forwarding nodes measure the node density based on the neighboring nodes $(R)$ available in the routing cache. The node density of proximity nodes at primary node is denoted as $N(t)$, which can be computed using neighbor table. The node density can be computed as:

$$
N(t)=R-I N
$$

Where $I N$ is the number of nodes, which are unable to satisfy the following constraint:

$$
Q_{i}(t)>Q_{\min } \text { and } E_{i}(t)>E_{\min }
$$

\subsection{Marginal cost function}

In this approach authors aim to substantially reduce routing expenses leaving out any corresponding significant increase in route latency as well as request-zone. The approach proposed avoids considerable rebroadcasting, delay in query at intermediate nodes and broadcast-repealing. One assumption for implementing the approach is that the attempt interval must be long enough so that the query is forwarded $h+\delta^{(i)}(t) * h$ hops away. The limit $\delta^{(i)}(t) * h$ is introduced to act as a factor of marginal cost to limit the extent of the search. This means that larger the value of $\delta^{(i)}(t)$ larger will be the search area. So, an ideal value needs to be determined which means a minimized search region. Let $\delta^{(i)}(t)$ be the marginal cost at $i^{t h}$ primary node at time $t$. It can be formulated as follows:

$$
\delta^{(i)}(t)=\frac{M_{a v g}^{(i)} * w_{1}+E_{a v g}^{(i)} * w_{2}+Q_{a v g}^{(i)}(t) * w_{3}+N^{(i)} * w_{4}}{M_{a v g}^{(i)}+E_{a v g}^{(i)}+Q_{a v g}^{(i)}(t)+N^{(i)}} \quad \delta \in(0,1)
$$

The multiplication factor $\delta^{(i)}(t)$ is a combined metrics of factors affecting mobility, energy, nodestatus and node-density. The factor $\delta^{(i)}(t)$ is then normalized to obtain value within the standard range $(0,1)$. The variables $M_{\text {avg }}^{(i)}, E_{\text {avg }}^{(i)}, Q_{\text {avg }}^{(i)}(t)$ and $N^{(i)}$ are used to denote the metrics average mobility, average energy, average node-status and node-density respectively which are actually determined by the $i^{t h}$ primary node available on old-route. In Eq. 9, $M_{\text {avg }}^{(i)}$ denotes impact of mobility on expanding request-zone in route-discovery, which can be determined as:

$$
M_{\text {avg }}^{(i)}=D_{\text {avg }}^{(i)} * w_{11}(t)+S_{\text {avg }}^{(i)} * w_{12}(t)+V^{(i)}(t) * w_{13}
$$

Different factors have different levels of contribution. The weights ${ }^{1} w 1, w 2, w 3, w 4$ in Eq. 9 and $w_{11}, w_{12}, w_{13}$ in Eq. 10 denote the relative importance of the metrics used in the cost functions. As the weights are not variables, these need to be determined carefully and wisely. The intermediate nodes that were part of the previous route become primary nodes. The weights are assigned following this process: for the duration when intensity of energy and mobility of nodes around primary nodes is moderate in measure, weight assigned mobility as well as to energy is higher than the node density. The reason for this is the fact that the target node would be in the vicinity of the primary node. On the contrary, higher weight is assigned to node density factor in case there is need to increase the search region. These weighing factors are computed in AHP process that gives the optimal weights. In this case, the value of $\delta^{(i)}(t)$ is determined only using the proximity information of primary nodes, which keep on passing the query ahead within the

\footnotetext{
$1 w_{1}+w_{2}+w_{3}+w_{4}=1$ and $w_{11}+w_{12}+w_{13}=1$
} 
given limit and high probability of discovering new route. The additional limit gives a marginal cost of forwarding a message from $h$ ring to $\delta^{(i)}(t) * h$ as in equation 11 .

$$
\text { marginal cost }=\sum_{i=1}^{\delta^{(i)}(t) * h} n_{i}
$$

Authors for the better understanding, retain the term definitions in [19], hence define neighbourhood as hopewise distance whereas search span as request - zone. The use of request-zone in approach explained refers to the query being forward by the previous path nodes $(h)$ encompassing source as well as target node spanning to include extended region 11. So the total number of queries can be calculated as

$$
Q=h+\underbrace{k+\sum_{i=1}^{\delta^{(i)}(t) * h} n_{i}}_{\text {Extended Search Region }}
$$

\subsection{Working process of SBA}

Following Figure 4, all the nodes shown in black color are old route-nodes, which have been identified in earlier communications. To initiate route discovery by the source node, cache is used to move the query forward. As query is passed along, visited node takes decision on increasing hop-count based on the two conditions: (a) Whether node itself is in old-route available in the header of query, and (b) Whether the hop-count value of the query has reached its upper limit. If both conditions are false, current node increases the hop-count before forwarding query. If both the conditions are true, nodes carry on with query forwarding business without incrementing hop-count. Otherwise the old route-nodes declare themselves as primary nodes if attempt seems failed. Thus we obtain primary and secondary nodes, which are explained as follows:

Primary node: The nodes on old routes where counter reaches to upper limit declare themselves as primary nodes. This case arises when the target node has relocated thus becomes unreachable. In this case, secondary nodes forward the query in expanded request-zone. These nodes are shown in black color in Figure 4.

Secondary node: The ordinary nodes including old route-nodes where counter is less than upper limit are referred as secondary nodes. This is the case where present node is either unvisited node or old route node lying on predefined request-zone. These nodes are shown in red color in Figure 4. To do so, secondary nodes pass the query ahead based on forwarding process.

\subsubsection{Utilizing most recent route for query-forwarding}

It has been researched that the new route discovered is not very distinct if we compare it to the route that has been used in most recent past. In the forwarding approach based on algorithm being cache aware, route discovery makes the use of the previous route and chooses the set of nodes as $R_{\text {old }}$ for query-broadcast. These are defined as old route-nodes. The query traverses these nodes with high likelihood of $n$ nodes that were part of old route no longer fulfil our requirements. Out of those, $k$ or more ordinary nodes may replace $k$ nodes to meet the current requirements. These $k$ nodes are shown by blue colored nodes in Figure 4 . Thus the process of passing query forward continues based on the locality and message to notify this is sent to the source. Secondary nodes broadcast query to its neighboring nodes. On receiving the query, all ordinary nodes will raise the counter of hope-count by 1 before broadcasting it to its neighboring nodes. These increments are shown at red colored nodes in Figure 4. It continues till the upper limit to the count is reached. 
If the query is received by an old route-node in predefined request-zone, the counter increment doesn't take place and the query is forwarded.

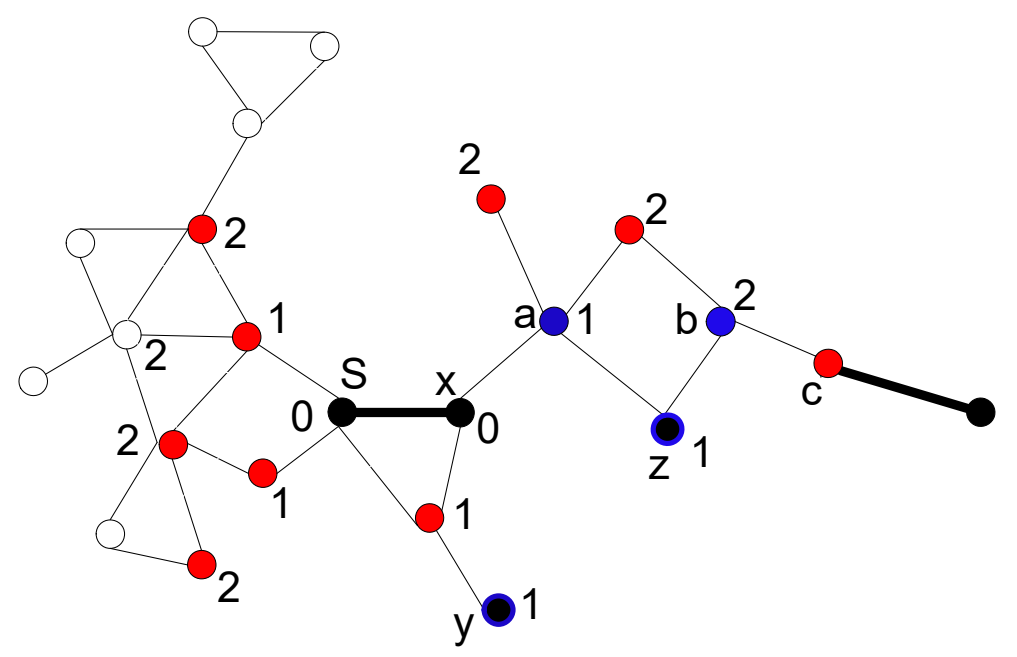

Fig. 4: Context assisted query-broadcast in SBA

\subsubsection{Reactive query-broadcast at primary nodes}

In the research efforts so far, the source node is expected to make multiple attempts for successful discovery of the route. In case the target node is found unreachable, a request for on demand zone expansion is raised. This is marginal increase and its based on the opinion that the target-node would not have shifted very far off in a given span of time and its likely to find in a little expanded request zone. Keeping this in mind the primary nodes compute the value of expansion based on $\delta^{(i)}(t)$. A desirable optimal value is computed based on the concept that lower weights are to be assigned to energy and movement if a node has low energy and high movement (see equation 9). With continued expansion requests based on target moving further, a number of nodes gets higher weight in comparison to energy and pause-time. Otherwise in order to reduce the request zone, $\delta^{(i)}(t)$ is made to shrink and more weight is assigned to energy and pause time. For this expansion, primary nodes are responsible, which are shown in black colored nodes with blue circles in Figure 4.

The couple of approaches discussed above handle to the request for zone expansion differently. While cache awareness based forwarding follows notion of $k$ value as used in literature[19]. On the other hand, On demand broadcast of the query exploits the node attributes.

\subsection{AHP assisted expanding request-zone}

AHP [12] provides a base to solve the multi-criteria decision making (MCDM) processes. Several applications of AHP have been noticed such as prescription of medicines, software development process, transportation and other sectors involving activity process [8,7]. Doctors can use AHP to implement the automatic prescription of medicines based on the cost, quality, patient condition, etc. Such activities have also been noticed in ad-hoc networks where an optimal route and querybroadcast are always desirable. Different metrics, for example hop-count, delay, query-transmission etc., and node attributes, for example energy, node density, mobility etc., are used in analyzing 
the network graph, which are further used in choosing the best path in route-discovery process. Several literatures like $[17,13]$ have used combined metrics based on node attributes multiplying with randomly chosen weighing factors. It would be better approach if AHP is applied on these criteria to get improved QoS of routing protocols.

\subsubsection{Procedural steps of AHP}

Here, AHP is applied in order to estimate weighing factors for the given equation 9 . The procedural steps of AHP method is detailed below:

Step 1. Define hierarchical structure of MCDM problem: In this step, a complex MCDM problem is decomposed into a hierarchy of interrelated criteria chosen as decision elements. This hierarchical structure of the problem contains at least 3 levels including goal, criteria and alternatives as shown in Figure 5. This hierarchical structure may also be extended to further dividing the criteria into subcriteria between goal and alternatives.

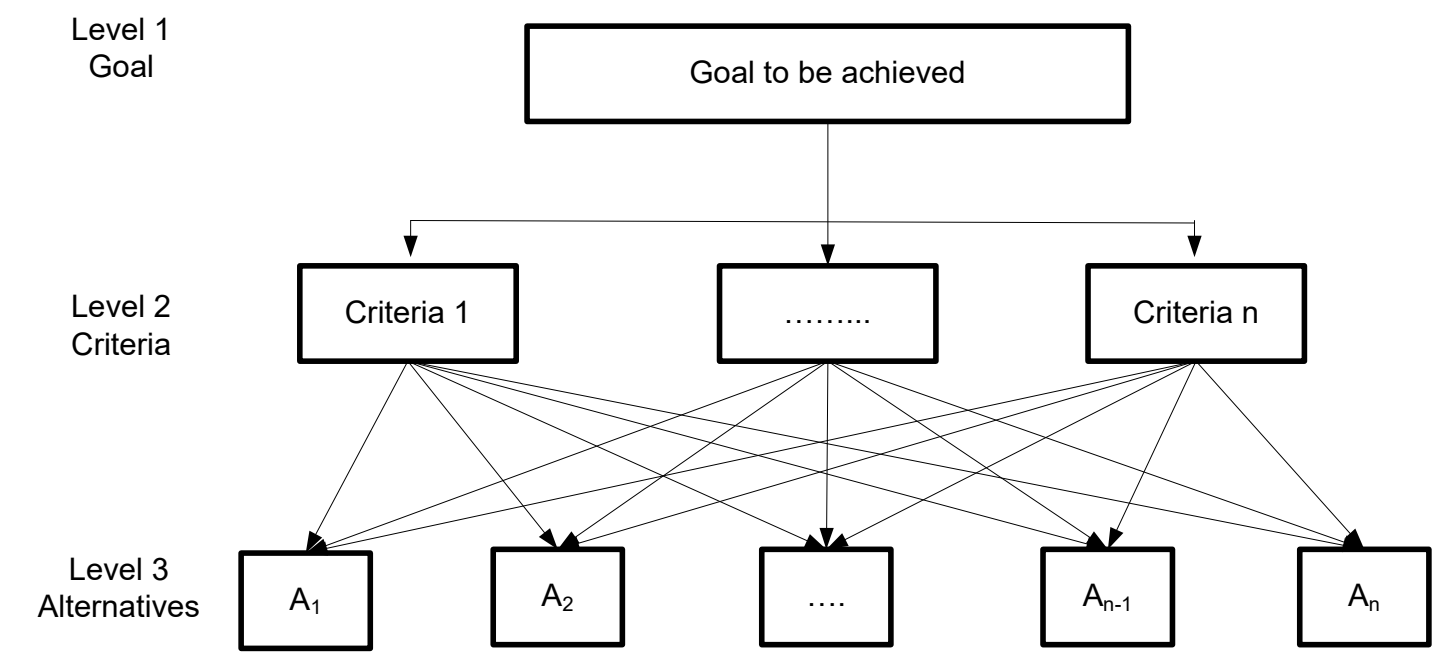

Fig. 5: General hierarchy of MCDM process

Step 2. Determine the pairwise comparisons for each criteria and alternatives: After determining the criteria and their relationship with the help of hierarchical structure in step 1 , comparison matrix is built to compute the the priority weights of the criteria. At each level, each

\begin{tabular}{ll}
$C_{i j}$ Intensity & Description \\
\hline 1 & i and $\mathrm{j}$ are equally preferable \\
3 & i is moderately preferable than $\mathrm{j}$ \\
5 & i is strongly preferable than $\mathrm{j}$ \\
7 & i is very strongly preferable than $\mathrm{j}$ \\
9 & i is extremely preferable than $\mathrm{j}$ \\
$2,4,8$ & Intermediate values between adjacent scales
\end{tabular}

Table 1: Scales for pair-wise comparison matrix 


\begin{tabular}{ccccccccccc}
\hline Size of the matrix (n) & 1 & 2 & 3 & 4 & 5 & 6 & 7 & 8 & 9 & 10 \\
Random Index (RI) & 0 & 0 & 0.58 & 0.9 & 1.12 & 1.24 & 1.32 & 1.41 & 1.45 & 1.49 \\
\hline
\end{tabular}

Table 2: Relationship between size of matrix and random consistency index

criterion is compared with other criteria based on their importance and level of influence. In AHP, this comparison is done with the help of a 9-point standardized comparison scale as detailed in Table 1. A pairwise comparison matrix is created for each criteria and its corresponding alternatives.

Assuming $C=\left\{C_{j} \mid j=1,2, \ldots, n\right\}$ is representing a set of criteria or decision elements of MCDM problem, where $\mathrm{n}$ is number of criteria. Each element of the comparison matrix $\mathrm{A}, \mathrm{ie}, a_{i j}(\mathrm{i}, \mathrm{j}=1$, $2, \ldots, n)$, represents its normalized relative weights as shown in Equation 13.

$$
A=\left(\begin{array}{cccc}
a_{11} & a_{12} & \cdots & a_{1 n} \\
a_{21} & a_{22} & \cdots & a_{2 n} \\
\vdots & \vdots & \ddots & \vdots \\
a_{m 1} & a_{m 2} & \cdots & a_{m n}
\end{array}\right)
$$

where $a_{i j}=1 / a_{j i}, a_{i j}>0$

The weight vector $w$ is determined using the characteristic equation, as shown in equation 14 .

$$
A w=\lambda_{\max } w
$$

where $\mathrm{A}$ is the pairwise comparison matrix of $n$ criteria, $w$ is the weight vector, and $\lambda_{\max }$ is the maximum eigenvalue the said matrix.

Step 3. Consistency Check of computed weights: In AHP, the pairwise matrix is said fully consistent if both consistency index (CI) and consistency ratio (CR) are as smaller as possible (less than 0.10 ). These two metrics can be computed using equations 15 and 16 respectively.

$$
\begin{gathered}
C I=\frac{\lambda_{\max }-n}{n-1} \\
C R=\frac{C I}{R I}
\end{gathered}
$$

Where $R I$ represents the random index of consistency as used in Equation 16. These are standard values defined based on the number of criteria, as listed in Table 2 . The value of CR should be less than 0.10 . The vector of priority weights are considered consistent if the computed value of CR is less than 0.10 , which concludes that matrix A is sufficiently consistent. In case of consistency failure, step 2 is repeated to improve the consistency.

\subsubsection{AHP assisted weights estimation in SBA}

Choosing a small enough extended request-zone is defined as MCDM which is decomposed into a hierarchy of interrelated decision parameters. A hierarchy of said problem with two levels of criteria and alternatives is graphically represented in Figure 6 . The high level decision factors are mobility, energy, node status and node-density which are kept at second level in the hierarchy. Further these factors are expanded to the next level defining sub-criteria. Different sets of nodes are kept at the lowest level of the hierarchy which plays the role of decision alternatives to extend the request zone. Each parameter in this hierarchy has relative importance with other parameters on choosing weighing factors through comparison matrix $C$ as given in equation. We have prepared a set of 
relative importance for mobility, energy, node-status and node-density after an exhausted study of different literature discussed in Section 2. The set for first level criteria is given as:

$$
\left\{C_{M E}, C_{M T}, C_{M N}, C_{E T}, C_{E N}, C_{T N}\right\} \Rightarrow\{3,7,9,5,7,3\}
$$

In this set, each notation is indicating the relative importance with others according to the scales defined for pair-wise comparison matrix as shown in Equations 17 and 18. For example, $C_{M T}$ indicates the mobility is extremely more preferable than node-density. Following the same scales, the set $\{1,5,0.3\}$ is defined for $C_{D S}, C_{D V}, C_{V S}$ indicating the relative importance between next level sub-criteria distance-speed, distance-variation, and variation-speed respectively. These two sets are used to compute the optimal weighing factors which are further used in combined weight metric given in equations.

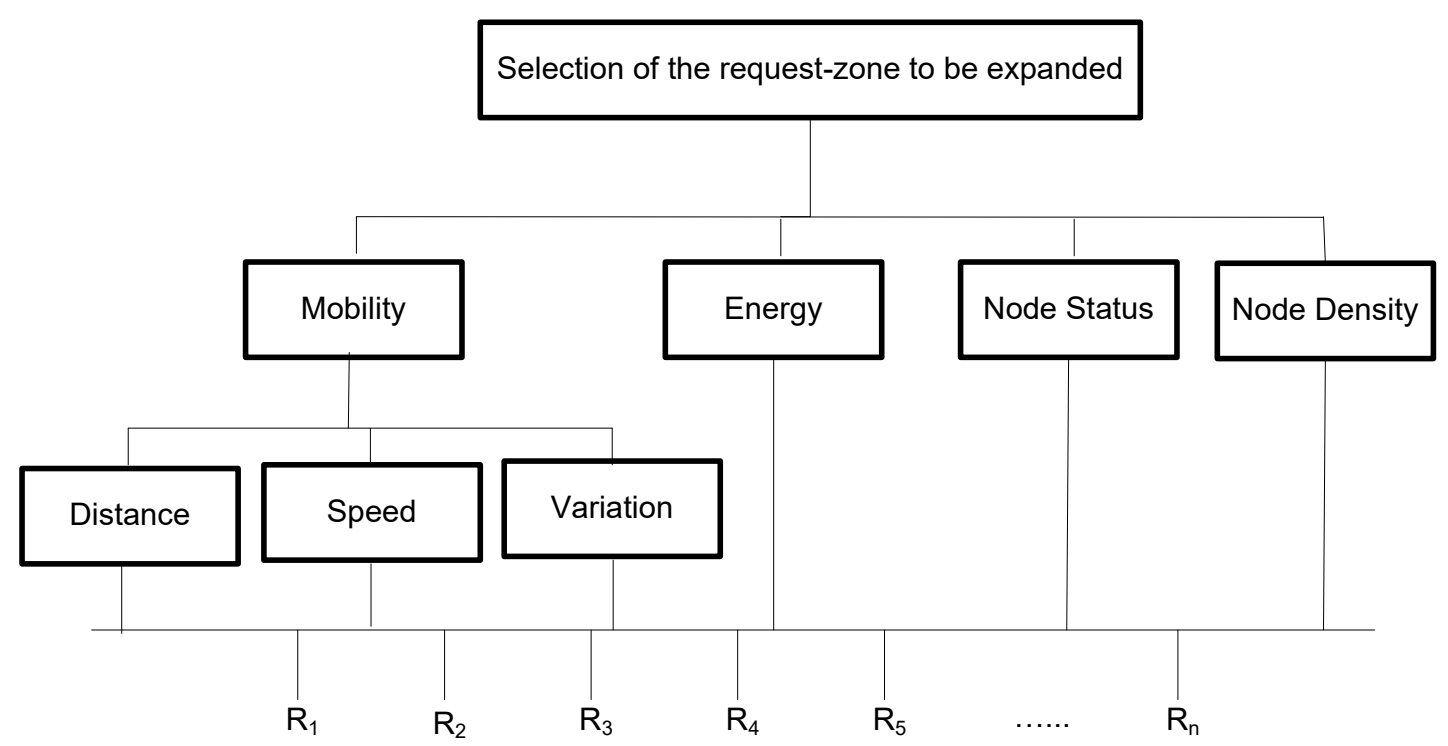

Fig. 6: Criteria of marginal factor for choosing tolerable extended search region

First level comparison matrix is given as:

$$
C_{1}=\left(\begin{array}{cccc}
1 & C_{M E} & C_{M T} & C_{M N} \\
C_{E M} & 1 & C_{E T} & C_{E N} \\
C_{T M} & C_{T E} & 1 & C_{T N} \\
C_{N M} & C_{N E} & C_{N T} & 1
\end{array}\right)
$$

Second level comparison matrix is given as:

$$
C_{2}=\left(\begin{array}{ccc}
1 & C_{D S} & C_{D A} \\
C_{S D} & 1 & C_{S A} \\
C_{A D} & C_{A S} & 1
\end{array}\right)
$$

AHP method is describe step by step in 3.4 that can also be studied from many literatures $[12$, $22,8,11]$. We have directly applied AHP method given in [12] from the lower levels to the upper one, for each criterion on each level, until a global weight vector is obtained. The computed weights for sub-criteria under mobility is given below: 


\begin{tabular}{llllll}
\hline Metric & Weights & Relative Raking & Local Metric & Local Weights & Local Ranking \\
\hline Mobility & 0.5738 & 1 & Distance & 0.4798 & 1 \\
& & & Speed & 0.4057 & 2 \\
& & & Variation & 0.1145 & 3 \\
Node Status & 0.0898 & 3 & & & \\
Energy & 0.2923 & 2 & & & \\
Node Density & 0.0441 & 4 & & & \\
\hline
\end{tabular}

Table 3: Scales for pair-wise comparison matrix

$$
\left(\begin{array}{c}
\text { Distance } \\
\text { Speed } \\
\text { Variation }
\end{array}\right) \Rightarrow\left(\begin{array}{c}
0.4798 \\
0.4057 \\
0.1145
\end{array}\right)_{\lambda_{\max }=3.0313, C I=0.0157, C R=0.0270}
$$

The computed weights of first level criteria is given below:

$$
\left(\begin{array}{c}
\text { Mobility } \\
\text { Node- status } \\
\text { Energy } \\
\text { Node }- \text { density }
\end{array}\right) \Rightarrow\left(\begin{array}{l}
0.5738 \\
0.0898 \\
0.2923 \\
0.0441
\end{array}\right){ }_{\lambda_{\max }=4.2600, C I=0.0867, C R=0.0963}
$$

In order to calculate the global weights of sub-criteria, we have multiplied each local weight by weight of mobility. Finally the overall ranking for all individual criteria is derived by comparing their relative priority which is given in Table 3. From Table 3, it is observed that mobility is highly desirable criteria and gets more importance than other metrics. We get Equations 19 an 20 after putting the value of weights in Equations 9and 10.

$$
\begin{gathered}
\delta^{(i)}(t)=\frac{0.5738 * M_{a v g}^{(i)}+0.2923 * E_{a v g}^{(i)}+0.0898 * Q_{a v g}^{(i)}(t) *+0.0441 * N^{(i)}}{M_{a v g}^{(i)}+E_{a v g}^{(i)}+Q_{a v g}^{(i)}(t)+N^{(i)}} \\
M_{a v g}^{(i)}(t)=0.4798 * D_{a v g}^{(i)}(t)+0.4057 * S_{a v g}^{(i)}(t)+0.1145 * V^{(i)}(t)
\end{gathered}
$$

Multiplying factor $\delta^{(i)}(t)$ is computed based on the status of the nodes' attributes lie in the proximity of primary nodes. For instance, assumed that the average speed and average energy of 10 neighboring-nodes are $5 \mathrm{~m} / \mathrm{s}$ and 70 units respectively. These nodes are experiencing average number of packets 5 in queue as network work load representing node status. With this information of neighboring-nodes, primary node computes the value of delta, which is 0.4121 . primary node would contribute approximately $40 \%$ of actual TTL-value for marginal cost.
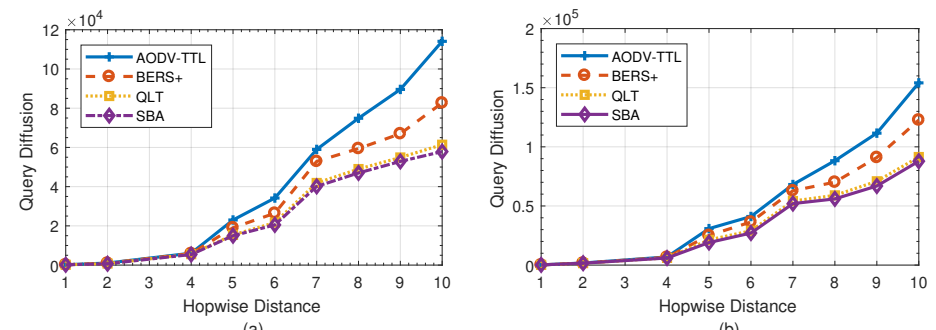

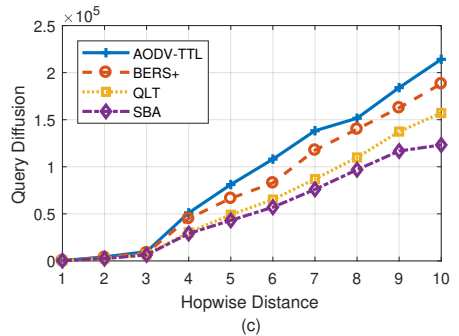

Fig. 7: Query broadcast for (a) low speed and (b) average speed (c) high speed 

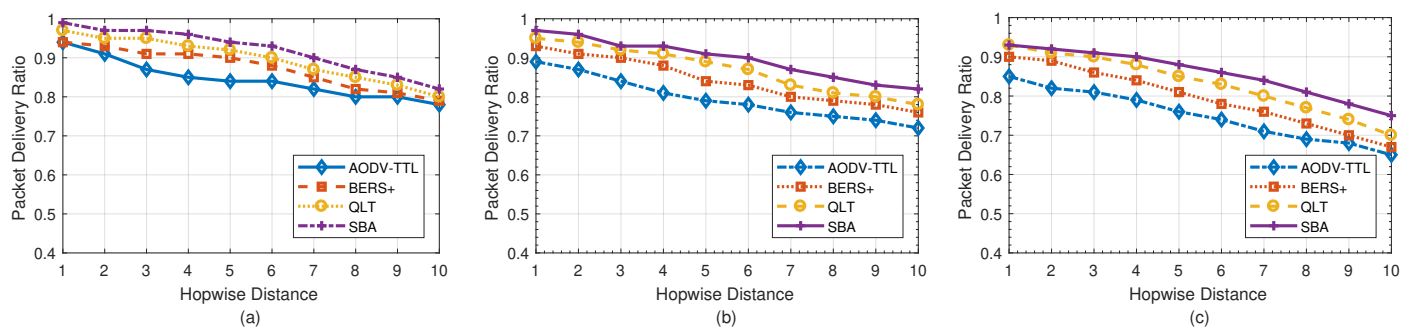

Fig. 8: Packet Delivery Ratio for (a) low speed and (b) average speed (c) high speed

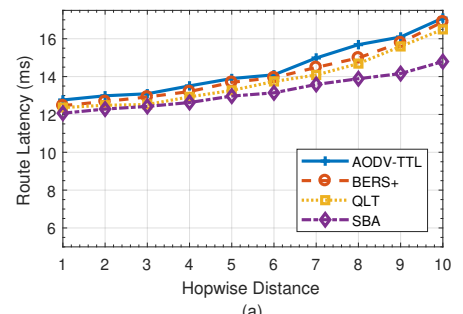

(a)

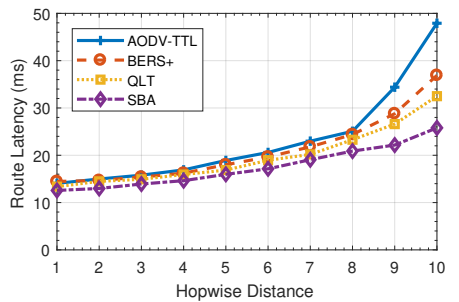

(b)

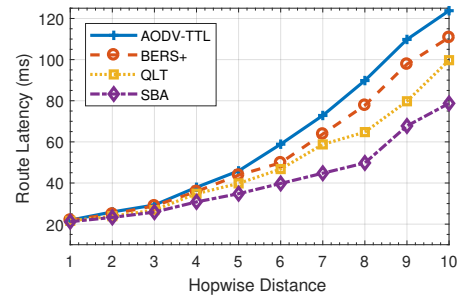

Fig. 9: Route Latency for (a) low speed and (b) average speed (c) high speed

\section{Simulation Setup and related performance metrics}

We have used NS2 to validate the performance of proposed approach, which is an event-driven network simulator and widely adopted by research community. The proposed approach and other related approaches (i.e. AODV-TTL, BERS+, QLT) are used as ad-on to built-in AODV of simulator for mobile nodes. Each-node is equipped with network layer events like packet-queuing, queryprocessing etc. Since primary node computes $\delta$ for expanding request-zone. It takes $(\approx 0.00031 \mathrm{~ms})$, which is negligible time and does not add any routing overhead in the route-discovery.

This simulation consists of 500 mobile nodes placing in a rectangular region of $1500 \mathrm{~m} \mathrm{x} 1500 \mathrm{~m}$. In this dynamic topology, node-distribution is non-uniform and all nodes follows the random walk mobility model. This model guides mobile nodes in choosing direction, speed and distance to be travelled in a defined region. This random movement of nodes generates new topology in which nodes are required to update their routing cache. This dynamic topology leads to link breaks thus causing route stale problem. Radio range of $120 \mathrm{~m}$ is set at each node to have the maximum distance between communicating nodes up to 12 hops. Here three mobility scenarios has been considered including low mobility, moderate mobility and high mobility. In low mobility scenario, nodes are allowed to travel with the speed of $3-4 \mathrm{~m} / \mathrm{s}$. While this speed is set $7-8 \mathrm{~m} / \mathrm{s}$ and $18-20 \mathrm{~m} / \mathrm{s}$ for moderate mobility and high mobility respectively. The distance between nodes is exponentially distributed with a mean of $5 \mathrm{~m}$ and nodes may in uniform direction within $\left[-45^{\circ},+45^{\circ}\right]$ with respect to the direction of the previous movement. Each node is equipped with battery energy of 1000 joules. The size of the communicated message is 512 bytes long, with 200 ms propagation delay. The size of the window is set 50 at each node, which represents the queue-capacity to contain the 10 messages. Each link capacity between two nodes is $10 \mathrm{Mbs}$. Following the said simulation set-up, all simulations are run for 900 seconds for different values of $\delta$ under low, moderate and high mobility. During simulation run, multiplying factor $\delta$ is calculated based on the present attributes of the proximity nodes of primary nodes, which is discussed in previous section. Here weights are taken 0.5738, 0.2923, 0.0898, and 0.0441 calculated using AHP method. 
In this simulation, the performance analysis is done based on 3 different performance metrics including number of query (query-diffusion), packet-delivery-ratio and route-latency. Query-diffusion represents the number of forwarding nodes lying in the both search-regions. Packet-delivery ratio is the proportion of number of packets received by the destination and total number of packets sent by the source-node. Route-latency is the average routing delay in finding the desirable route between two communicating nodes. It is measured as the time taken by the packet to reach the destination and going back to source-node.

\section{Results and Discussion}

All nodes are allowed to move in random direction with defined speed except two nodes including source and destination. These two nodes move in different directions during simulation run. At the start of the simulation, these communicating nodes are set at desirable positions and then move different directions thus increasing distance measured in hops. As it is observed that requestzone directly proportional to the distance between communicating nodes. The request-zone for farther nodes is needed large enough than request-zone of closer nodes, which poses the problem of link-breaks due to dynamic change in network topology. Thus it causes the route-maintenance overhead as distance between source and destination nodes increases. Based on this observation, all figures are plotted against the distance between source and destination nodes measured in hopcount. From the Figures 7, 8 and 9, obtained results can be observed in terms of said performance metrics in different mobility scenarios. The plots 7 depicts that proposed approach requires smaller request-zone comparative to other approaches AODV-TTL (traditional flooding), BERS+, QLT, thus reduces the query-broadcast significantly. The reason for providing smaller request-zone is single successful route-discovery attempt for moving communicating nodes in opposite directions, which is not in other approaches.

The plots 9 shows routing delay against the distance between two communicating nodes. In this plot, routing delay is lesser then other approaches because of lesser query-diffusion. Since communicating nodes are closer in the beginning, obtained result for proposed approach and QLT are very similar, but the the proposed approach reduces the routing delay significantly for larger distance. In addition to this, proposed approach also performs well comparative to AODV-TTL and BERS+. Routing delay in BERS+ is high because of added delay introduced at each participating node, while repeated broadcast increases the routing delay in AODV-TTL. Summary of the results for route-latency can be seen in the Figures 9(a), 9(b) and 9(c). For high mobile nodes, difference is high between the results of proposed approach and other approach for all performance metrics, which is shown in Figures 7 (c) and 9(c). Moreover highly dynamic topology leads to weak connections between nodes thus increasing number of route-maintenance is experienced.

As it is also observed that high mobile nodes leads to the weak connections between nodes, thus increasing number of route-discovery attempts. Moreover, such delay causes the less packet delivery ration as it is observed in Figure 8. This simulation favors the proposed techniques in improving the packet-delivery ratio. This is because of less congestion in the network that is reduced by proposed technique significantly.

\section{Conclusion and future directions}

This paper proposes cache-aware approach which overcomes some of the significant issues with route discovery in WANETs. The concern of research fraternity over high average latency, high consumption of power, network congestion giving rise to performance issues, have been dealt with in our proposed approach. Our methodology leverages routing cache in route discovery phase to confine the request-zone. The zone expands dynamically to determine optimal route successfully. 
To offset the overhead incurred in request-zone, suitable values of various parameters are obtained for successfully finding the route. Study reveals that technique proposed in the paper yields better results over various approaches discussed in sections of introduction and related work. In our simulation, we chose weights randomly to calculate the optimum value of $\delta$. In order to assign the priority to different node attributes, well known Analytical Hierarchy Process (AHP) has been applied. Simulation and Results sections reveal the output obtained with high efficiency.

The present study however has some limitations and points to some future directions as well. We have considered different previous contributions to assign the priorities and prepare the comparison matrix. It is also possible to conduct a survey and take the responses from the expert of related domain, which is not considered in our paper. In future, experts' responses can be considered for further study. Here we have prioritized a set of 4 metrics as criteria, which can also be increased to have more detailed study.

\section{References}

1. Ahmad, N., Hussain, S.Z.: Broadcast expenses controlling techniques in mobile ad-hoc networks: A survey. Journal of King Saud University-Computer and Information Sciences (2015)

2. Ahmad, N., Hussain, S.Z.: Analytical comparisons of query-broadcast repealing schemes in manets. Telecommunication Systems (TELS), Springer 70(1), 67-79 (2018)

3. Ahmad, N., Sethi, S.: An overview of query-broadcasting techniques in ad hoc networks. In: Mobile Computing. IntechOpen (2019)

4. Ahmad, N., Sethi, S., Ahmed, M.: Cache-aware query-broadcast to improve qos of routing protocols in manets Wireless Personal Communications pp. 1-18 (2020)

5. Al-Rodhaan, M.A., Mackenzie, L., Ould-Khaoua, M.: Improvement to blocking expanding ring search for manets. Dept of Computing Science, University of Glasgow, Glasgow, UK pp. 1-13 (2008)

6. Chang, N., Liu, M.: Revisiting the ttl-based controlled flooding search: Optimality and randomization. In: Proceedings of the 10th annual international conference on Mobile computing and networking. pp. 85-99. $\operatorname{ACM}(2004)$

7. Darko, A., Chan, A.P.C., Ameyaw, E.E., Owusu, E.K., Pärn, E., Edwards, D.J.: Review of application of analytic hierarchy process (ahp) in construction. International Journal of Construction Management 19(5), 436-452 (2019)

8. Deng, X., Deng, Y.: D-ahp method with different credibility of information. Soft Computing 23(2), 683-691 (2019)

9. Gargano, L., Hammar, M.: Limiting flooding expenses in on-demand source-initiated protocols for mobile wireless networks. In: Parallel and Distributed Processing Symposium, 2004. Proceedings. 18th International. p. 220. IEEE (2004)

10. Hussain, S.Z., Ahmad, N.: Minimizing broadcast expenses in clustered ad-hoc networks. Journal of King Saud University - Computer and Information Sciences (2017)

11. Kumari, N.D., Shylaja, B.: Amgrp: Ahp-based multimetric geographical routing protocol for urban environment of vanets. Journal of King Saud University - Computer and Information Sciences 31(1), 72 - 81 (2019)

12. Li, J., Yang, Y., Saaty, T.L., Guo, H.: Cultural ranking of countries using the analytic hierarchy process methodology. In: The International Conference on Natural Computation, Fuzzy Systems and Knowledge Discovery. pp. 949-963. Springer (2019)

13. Li, X., Lv, Z., Wang, P., Sun, M., Qiao, M.: Combination weighted clustering algorithms in cognitive radio networks. Concurrency and Computation: Practice and Experience p. e5516 (2019)

14. Liu, J., Jiang, X., Nishiyama, H., Kato, N.: Throughput capacity of manets with power control and packet redundancy. IEEE transactions on wireless communications 12(6), 3035-3047 (2013)

15. Ouchitachen, H., Hair, A., Idrissi, N.: Improved multi-objective weighted clustering algorithm in wireless sensor network. Egyptian Informatics Journal 18(1), 45-54 (2017)

16. Park, I., Kim, J., Pu, I., et al.: Blocking expanding ring search algorithm for efficient energy consumption in mobile ad hoc networks. In: WONS 2006: Third Annual Conference on Wireless On-demand Network Systems and Services. pp. 191-195 (2006)

17. Pathak, S., Jain, S.: A priority-based weighted clustering algorithm for mobile ad hoc network. International Journal of Communication Networks and Distributed Systems 22(3), 313-328 (2019)

18. Persis, D.J., Robert, T.P.: Review of ad-hoc on-demand distance vector protocol and its swarm intelligent variants for mobile ad-hoc network. IET Networks 6(5), 87-93 (2017)

19. Prabhavat, S., Narongkhachavana, W., Thongthavorn, T., Phankaew, C.: Low overhead localized routing in mobile ad hoc networks. Wireless Communications and Mobile Computing 2019 (2019) 
20. Pu, I.M., Shen, Y.: Enhanced blocking expanding ring search in mobile ad hoc networks. In: New Technologies, Mobility and Security (NTMS), 2009 3rd International Conference on. pp. 1-5. IEEE (2009)

21. S. Zeeshan, H., Naeem, A.: Cluster based controlling of route exploring packets in ad-hoc networks. In: Proceedings of the 2nd International Conference on Advanced Computing, Networking, and Informatics, ICACNI'14. vol. 28, pp. 103-112. Smart Innovation, Systems and Technologies,Springer Verlag (2014)

22. Saaty, T.L.: Decision making with the analytic hierarchy process. International journal of services sciences $1(1), 83-98(2008)$

23. Sivakumar, N.R., Al Garni, A.: Efficiency of weighted rough set model in proactive protocol using grid. In: 2018 1st International Conference on Computer Applications \& Information Security (ICCAIS). pp. 1-5. IEEE (2018)

24. Sivakumar, N.R., Alaraj, A., Kuppusamy, P., Krishnan, S.: New strategy of probabilistic flooding with fuzzy \& rough sets techniques. In: 2017 2nd International Conference on Anti-Cyber Crimes (ICACC). pp. 133-136. IEEE (2017)

25. Sun, W., Yang, Z., Zhang, X., Liu, Y.: Energy-efficient neighbor discovery in mobile ad hoc and wireless sensor networks: A survey. IEEE Communications Surveys \& Tutorials 16(3), 1448-1459 (2014)

26. Taha, A., Alsaqour, R., Uddin, M., Abdelhaq, M., Saba, T.: Energy efficient multipath routing protocol for mobile ad-hoc network using the fitness function. IEEE access 5, 10369-10381 (2017)

27. V. K. Quy, L.N.H., Han, N.D.: Ceprm: A cloud-assisted energy-saving and performance-improving routing mechanism for manets. Journal of Communications 14(12), 1211-1217 (2019)

\section{Appendices}

A List of abbreviations 


\begin{tabular}{ll}
\hline Study & Abbreviation \\
\hline TTL-ERS & TTL sequenced based ERS \\
MPR & Multi Point Relay Set \\
FNSB & Forward-node-set based broadcast in Clustered \\
ZRP & ZANETs \\
QLT & Query Localization Technique \\
FRESH & FResher Encounter SearcH \\
HoWL & Hop-Wise Limited broadcasting \\
LHBA & Limited Hops Broadcasting Algorithm \\
WRS & Weighted Rough Set based Broadcasting \\
PQB & Power-aware query broadcast \\
DWCA & Distributed Weighted Clustering Algorithm \\
BERS & Blocking Expanding Ring Search \\
BERS* & Enhance Blocking Expanding Ring Search \\
ACRR & Aodv Controlled Route Request \\
IRS & Inreasing Ray Search for efficient query broadcast \\
MWCDB & Minimum weight connected dominating set based \\
BERS+ & broadcast \\
PAQB & Improved Blocking Expanding Ring Search \\
ORPF & Probabilistic approach for query broadcast \\
BCIR & Optimize route-discovery and packet forwarding \\
tBERS* & Broadcast Cancellation Initiated on Resource \\
TSBERS & time-efficient BERS (tBERS), time-efficient BERS* \\
ABCQM & Two sided expanding ring search \\
eBERS & Adaptive Broadcast Cancellation Query Mechanism \\
MBERS+ & Energy-efficient BERS \\
PFFRS & Modified-BERS \\
FFQB & Probabilistic flooding with fuzzy \& rough sets \\
BCQB & Fitness function based query-broadcast \\
\hline &
\end{tabular}


Figures

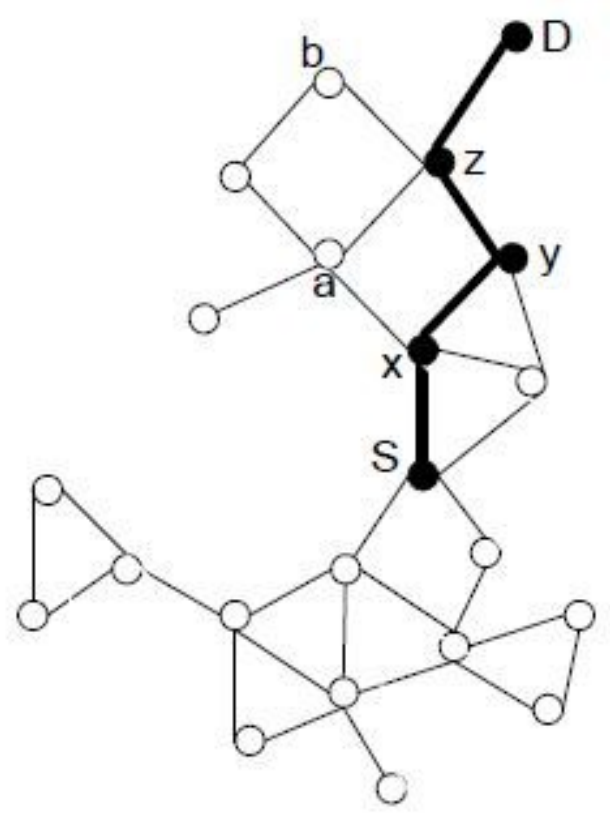

(a)

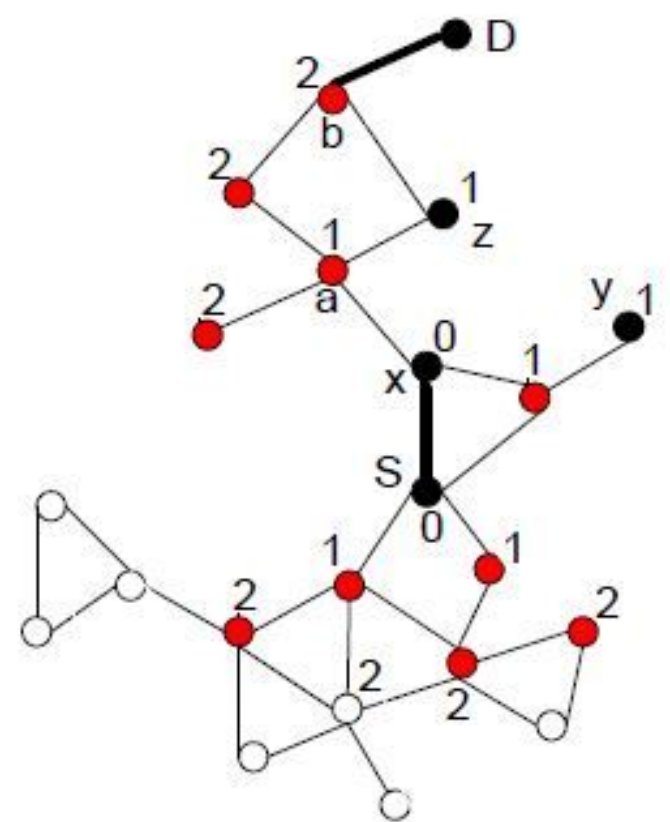

(b)

Figure 1

(a)Most recently used route. (b)Failed attempt of route discovery when target node or any of the old routenodes moves to new location. 


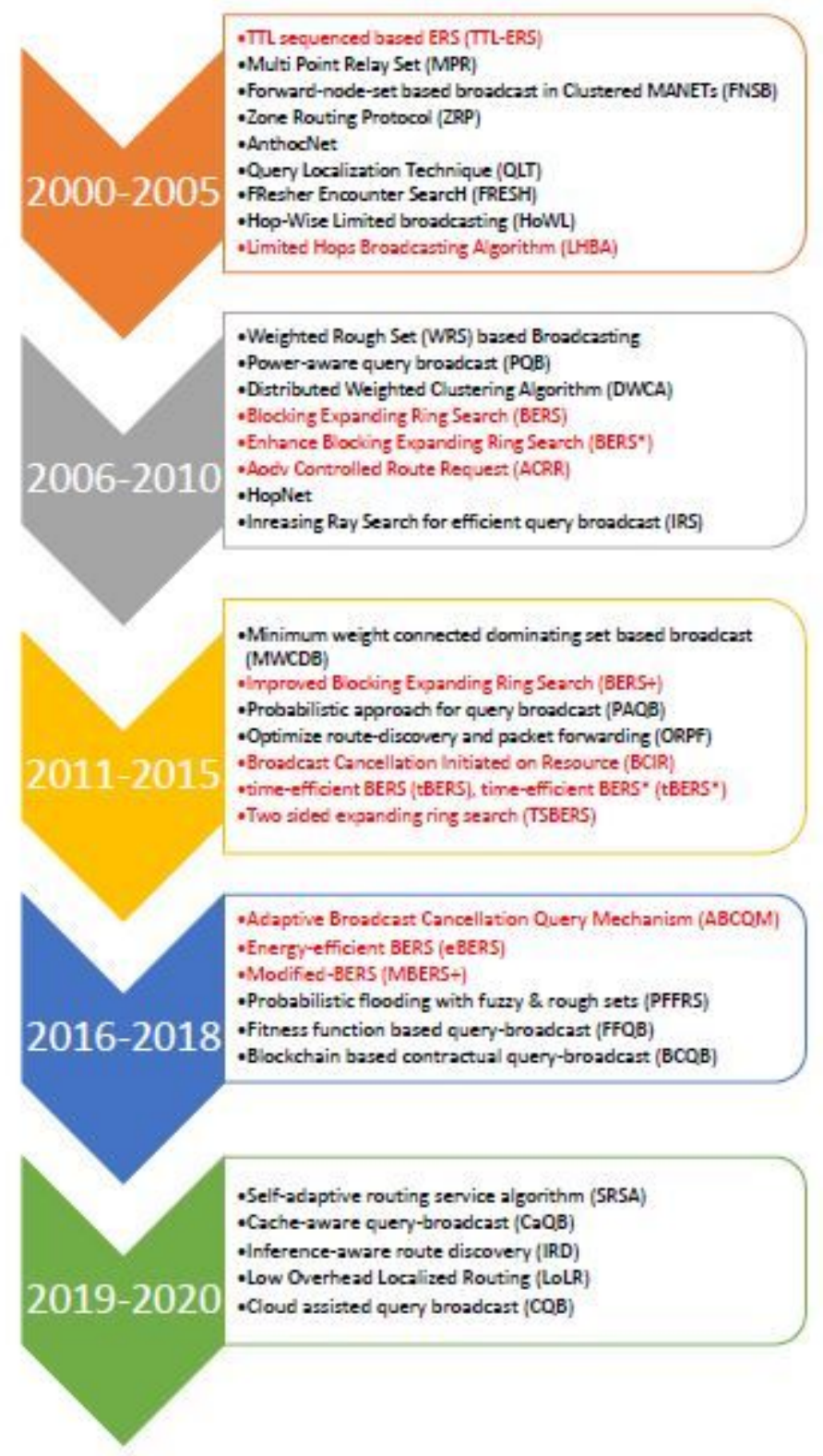

Figure 2

Classication of query-broadcast methods (Please see full abbreviations in appendix A) 


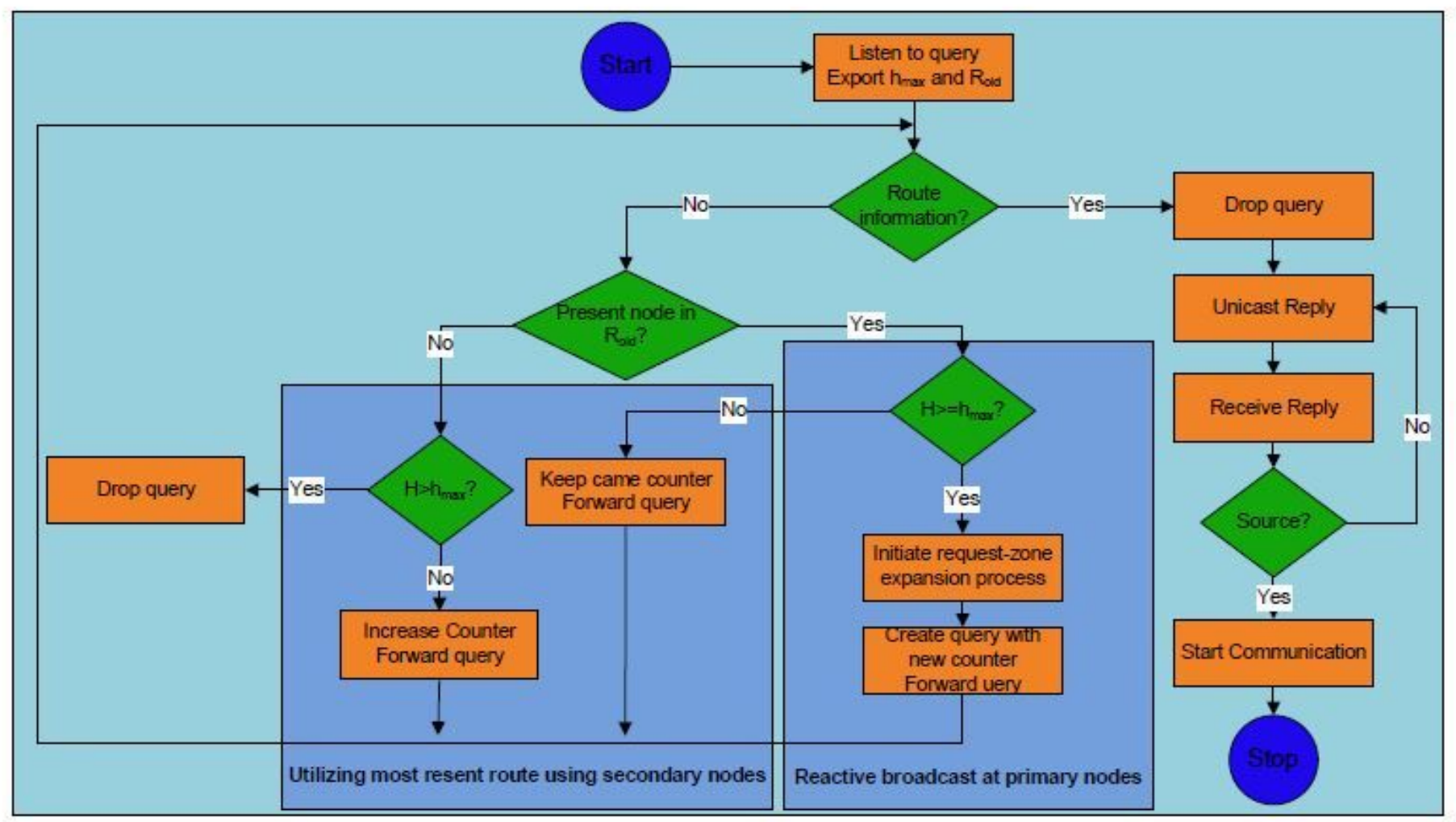

Figure 3

Work flow of proposed approach

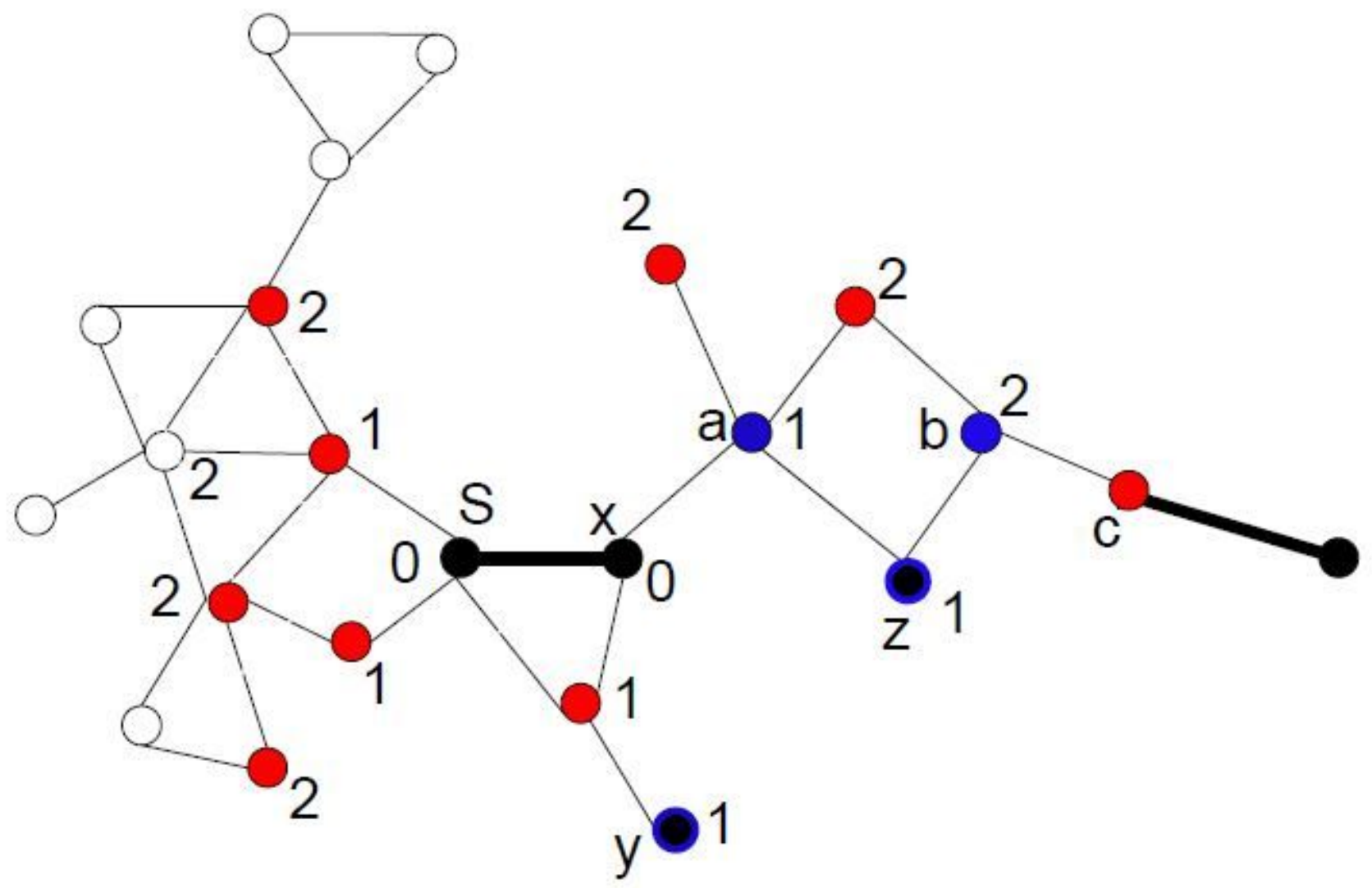


Figure 4

Context assisted query-broadcast in SBA

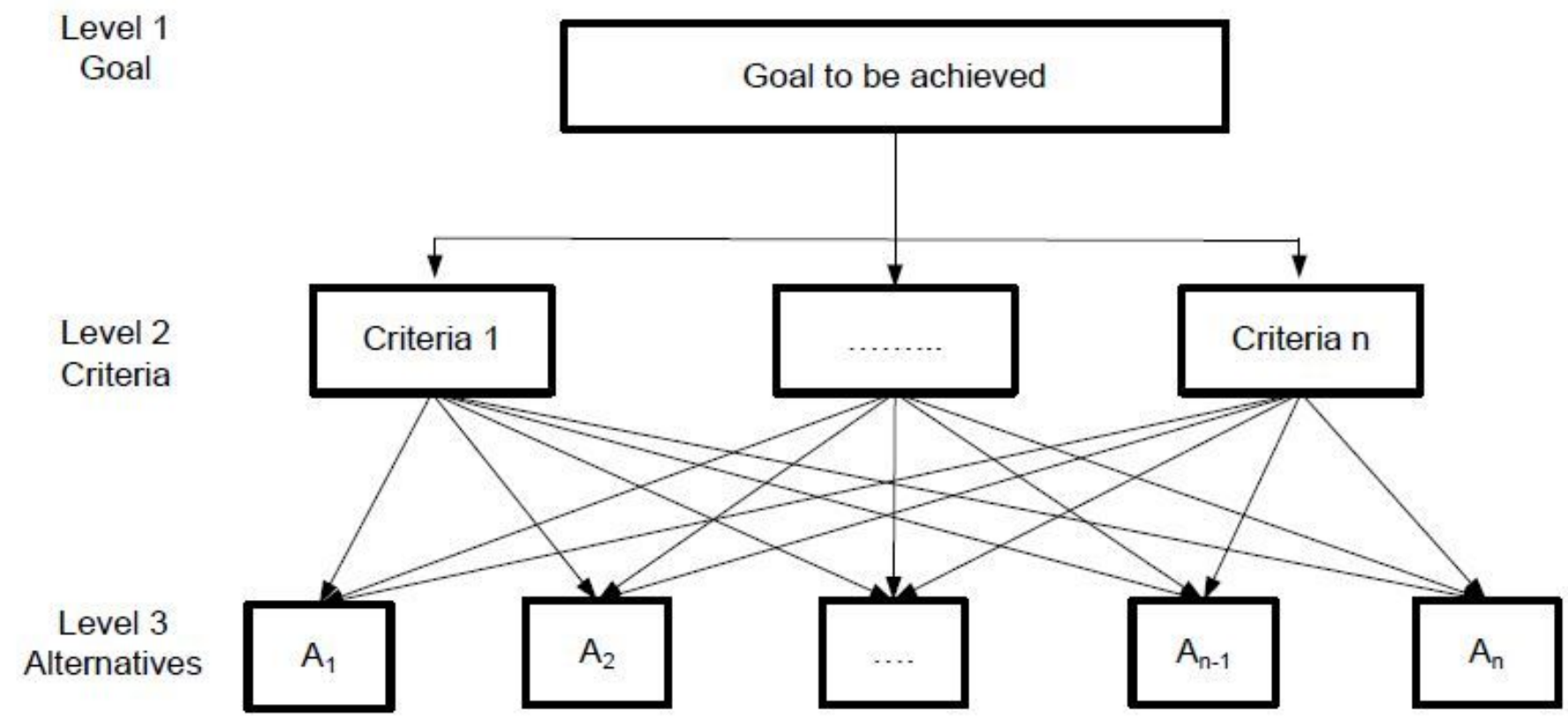

Figure 5

General hierarchy of MCDM process

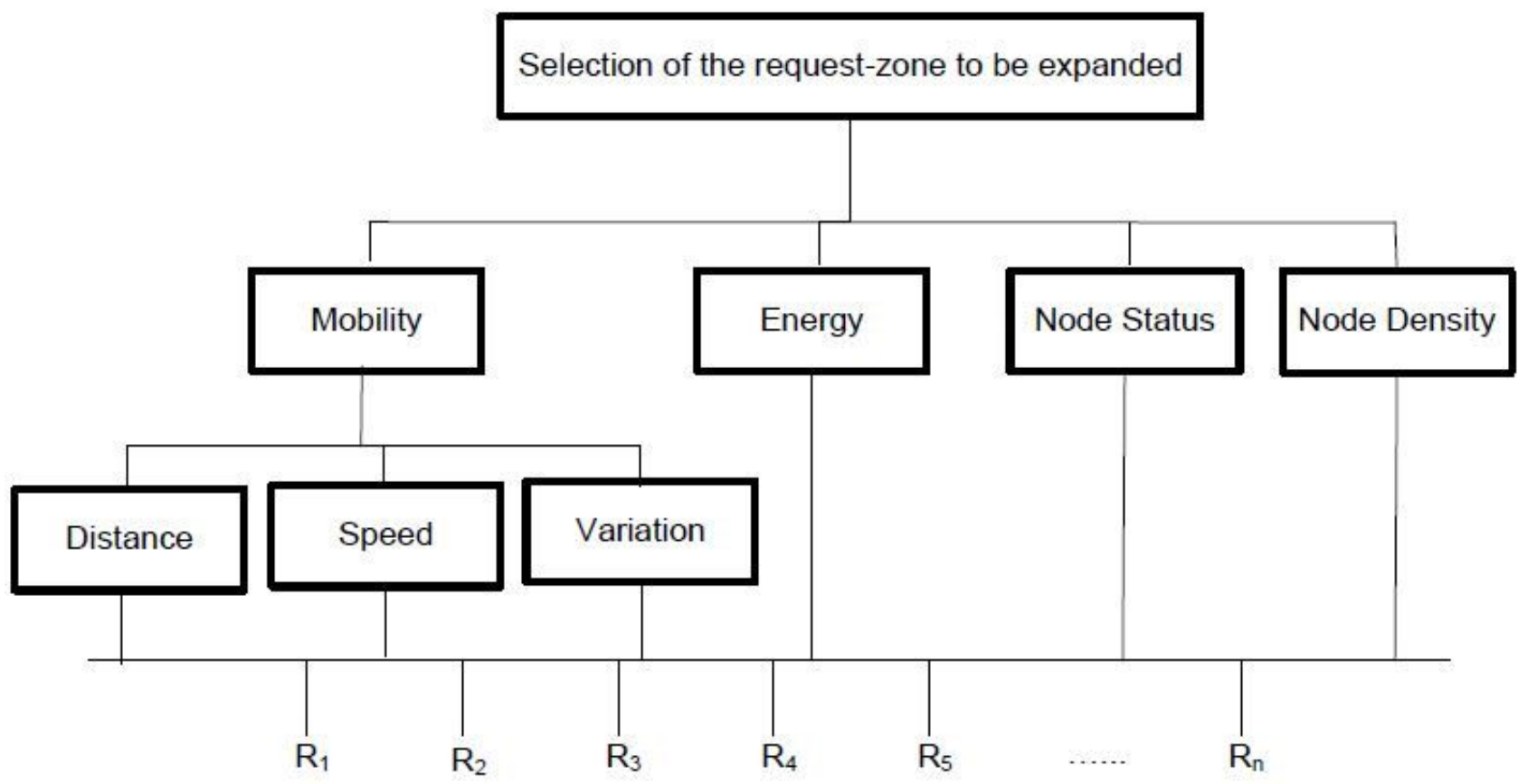

Figure 6

Criteria of marginal factor for choosing tolerable extended search region 


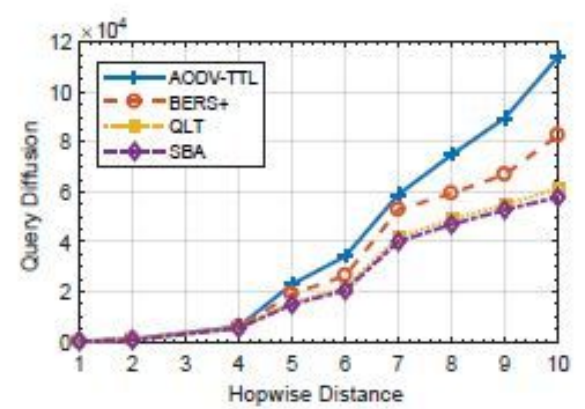

(a)

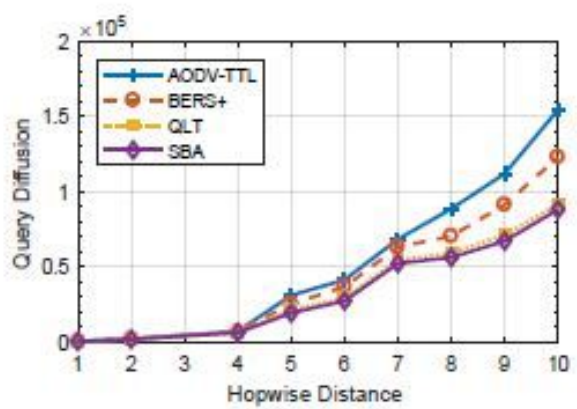

(b)

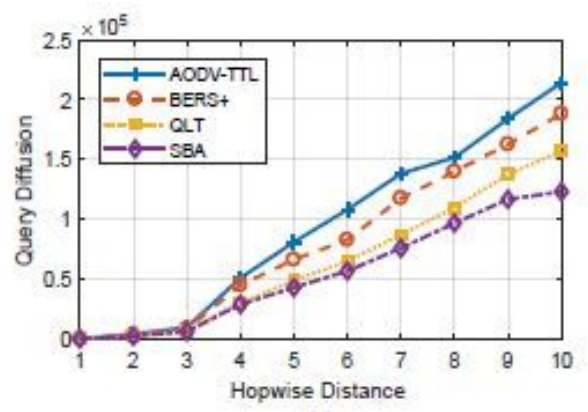

(c)

\section{Figure 7}

Query broadcast for (a) low speed and (b) average speed (c) high speed

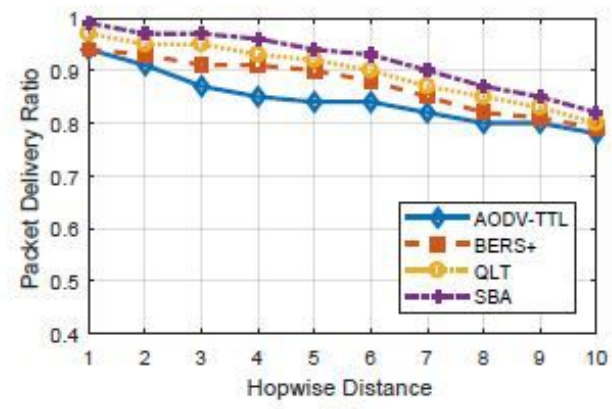

(a)

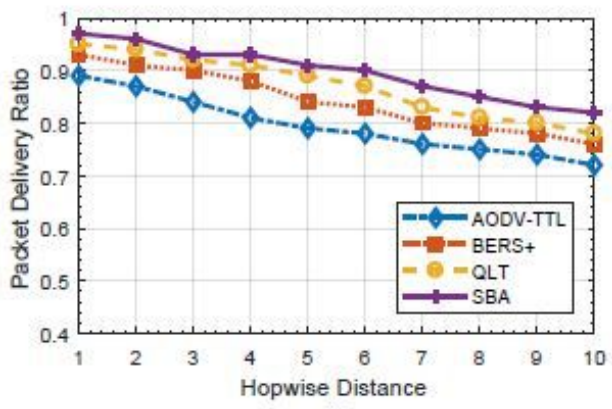

(b)

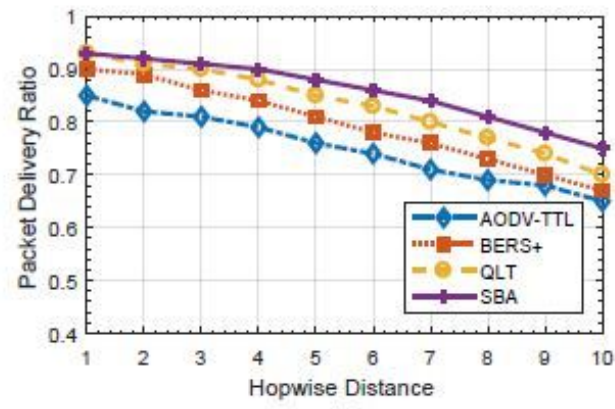

(c)

Figure 8

Packet Delivery Ratio for (a) low speed and (b) average speed (c) high speed

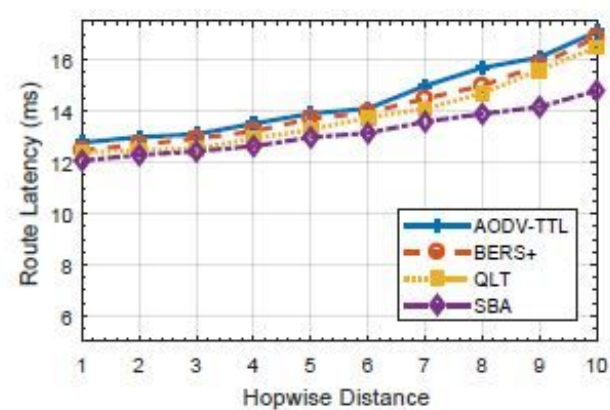

(a)

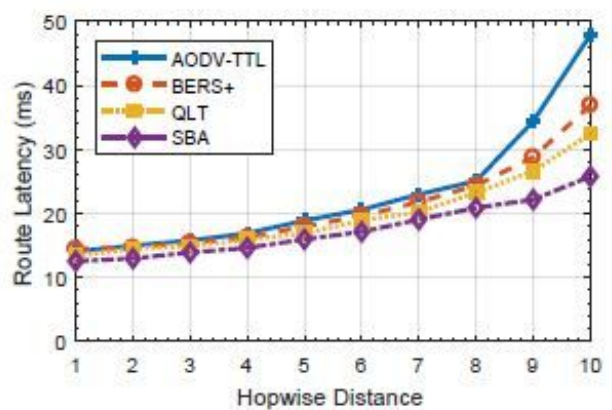

(b)

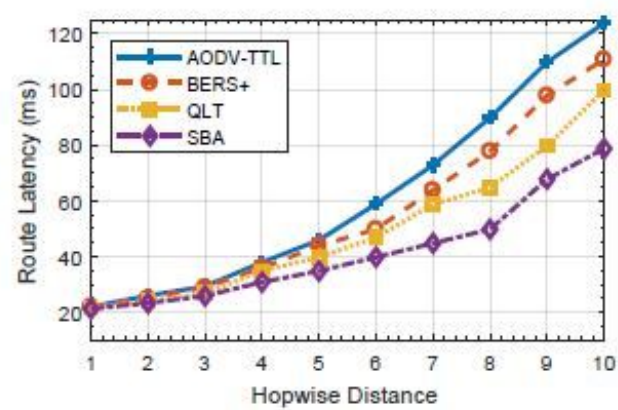

(c)

Figure 9

Route Latency for (a) low speed and (b) average speed (c) high speed 